Sir,

\section{Novel software to self-monitor central serous retinopathy}

Central serous retinopathy (CSR) is a rare condition associated with endogenous hypercortisolism, involving detachment of the sensory retina and typically affecting males aged 20-50 years. It is characterised by a central scotoma, reduced visual acuity and metamorphopsia. Here, we present the case of a patient with CSR who developed a computer programme to chart the course of his scotoma.

\section{Case report}

A 64-year-old man presented with reduced visual acuity, distortion, and a central positive scotoma in his right eye. These symptoms had worsened over the preceding month. Following investigations, including fundus fluorescein angiography which showed an ink-blot pattern, he was diagnosed with central serous retinopathy (CSR). CSR is a condition of uncertain aetiology, characterised by the detachment of the sensory retina due to fluid leakage from a hyperpermeable choriocapillaris. ${ }^{1}$

Since the onset of his symptoms, the patient had used his skills as a computer engineer to document the changing size of his scotoma. He did this by comparing his visual field defect with a circle of known diameter on a computer screen. The distance between his eyes and the screen when the on-screen circle matched the scotoma was then used to calculate the solid angle subtended by the scotoma. Solid angle essentially measures how large the scotoma appears to the patient.

He was encouraged to continue this following diagnosis, and his results after 98 days are presented here (Figure 1). No treatment was given during this time.

\section{Comment}

To our knowledge, this is the first instance of a patient charting the course of his CSR. This method of

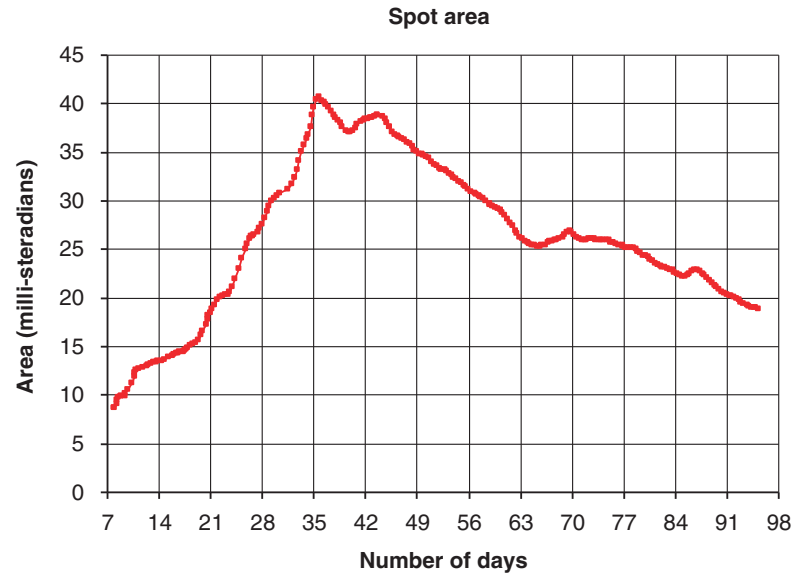

$\rightarrow$ Area (weighted moving avg)

Figure 1 Change in area (solid angle) of the patient's scotoma over time. measurement represents a simple way of closely monitoring any change of the CSR-associated scotoma. Further investigation is warranted into whether this pattern is repeated in other cases, or if it varies between patients.

Clinical applications of frequently measuring the size of the scotoma would result from being able to assess the impact of CSR on vision and monitor any improvement or worsening. Several non-invasive therapies have been suggested for CSR; ${ }^{2-4}$ this method of measuring scotomata could be used in comparing therapeutic options. It may also assist when deciding whether to treat, rather than basing this just on the duration of the episode. Currently, treatment with argon laser photocoagulation is considered after 4 months of persistent CSR. ${ }^{5}$ However, if after this time there was spontaneous reduction of the scotoma, intervention could be avoided.

Detailed charting of the change in scotoma size may also help determine the associations between CSR and other factors which remain unclear, for example, hypercortisolic states.

The computer program designed by this patient, 'CSR utility', is currently freely available to clinicians and patients at http://www.gpsu.co.uk/csru/

An example of how to use the program is available to download from http://www.gpsu.co.uk/csru/ mmcsr.html

\section{References}

1 Spitznas M. Pathogenesis of central serous retinopathy: a new working hypothesis. Graefe's Arch Clin Exp Ophthalmol 1986; 224: 321-324.

2 Pikkel J, Beiran I, Ophir A, Miller B. Acetazolamide for central serous retinopathy. Ophthalmology 2002; 109(9): 1723-1725.

3 Tatham A, Macfarlane A. The use of propanolol to treat central serous chorioretinopathy: an evaluation by serial OCT. J Ocul Pharmacol Ther 2006; 22(2): 145-149.

4 Nielsen JS, Weinreb RN, Yannuzzi L, Jampol LM. Mifepristone treatment of chronic central serous chorioretinopathy. Retina 2007; 27(1): 119-122.

5 Kanski JJ. Central serous retinopathy. In: Clinical Ophthalmology: A Systematic Approach, 5th edn. Butterworth-Heinemann: London, 2003, pp 421-423.

AMJ Turnbull', MS Abu-Ain², SC Lash², AS Murphy and AJ Lotery ${ }^{1,2}$

${ }^{1}$ University of Southampton, School of Medicine, Southampton General Hospital, Southampton, Hampshire, UK

${ }^{2}$ Southampton Eye Unit, Department of Ophthalmology, Southampton General Hospital, Southampton, Hampshire, UK

E-mail: abuain@hotmail.co.uk

Eye (2010) 24, 179; doi:10.1038/eye.2009.40; published online 27 February 2009 